\title{
Dental Anxiety: Interprofessional Research into an Intractable Global Problem
}

ISSN: 2637-7764

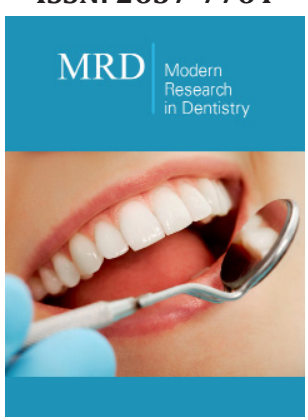

*Corresponding author: Zac Morse, Department of Oral Health, School of Clinical Sciences, Auckland University of Technology, 90 Akoranga Drive, Northcote, Auckland, 0627, Aotearoa, New Zealand

Submission: 阱 November 28, 2020

Published: 啙December 18, 2020

Volume 6 - Issue 1

How to cite this article: Zac Morse*, Keith Tudor. Dental Anxiety: Interprofessional Research into an Intractable Global Problem. Mod Res Dent. 6(1). MRD. 000626. 2020

DOI: $10.31031 /$ MRD.2020.06.000626

Copyright@ Zac Morse, This article is distributed under the terms of the Creative Commons Attribution 4.0 International License, which permits unrestricted use and redistribution provided that the original author and source are credited.

\section{Zac Morse ${ }^{1 *}$ and Keith Tudor ${ }^{2}$}

${ }^{1}$ Professor, Department of Oral Health, School of Clinical Sciences, Auckland University of Technology, Auckland, Aotearoa New Zealand

${ }^{2}$ Professor, Department of Psychotherapy \& Counselling, School of Clinical Sciences, Auckland University of Technology, Auckland, Aotearoa New Zealand

\section{Abstract}

This article offers an initial mini-review of dental anxiety and identifies future directions for research into nonpharmacological, therapeutic approaches to addressing this significant health issue.

Keywords: Dental anxiety; Nonpharmacological; Therapeutic approaches; Interprofessional; Oral health; Psychotherapy; Eye movement desensitisation reprocessing

\section{Introduction}

Question: What do you get when you cross (or at least introduce) a professor of oral health with a professor of psychotherapy? Answer: A common interest in psychological health and dental anxiety. This mini-review arose from a meeting of the two authors and our obvious identification of dental anxiety as a focus for joint research and research supervision. We emphasise health before illness as we both agree that just as mental health is critical to general health [1,2], so there can be no general health without oral health [3].

\section{Review}

Dental anxiety and fear are a significant public health problem in Aotearoa New Zealand, affecting one in eight of the population. Global estimates of this form of anxiety are comparable in other countries [4,5]. Dental anxiety is a significant barrier to oral health services that adversely affects the oral health of affected individuals and is recognised to have a multifactorial aetiology [6]. However, to date, the standard response to and management of this health issue is predominantly pharmacological, with sedation and general anaesthesia being common. This practice is cumbersome, expensive and requires a high standard of care to be administered safely but does not provide a cure for the underlying anxiety or phobia. Also, patients with dental anxiety are perceived as complex and thus consume disproportionate healthcare time and resources. Moreover, given the higher rates of tooth decay, dental pain and treatments requiring general anaesthesia in both Māori and Pasifika populations in Aotearoa New Zealand, any interventions that reduce dental anxiety particularly in these populations would redress significant inequities in this aspect of this country's population health.

Nonpharmacological responses and interventions for dental anxiety and phobia have included hypnosis and hypnotherapy [7]; eye movement desensitisation reprocessing (EMDR) [8]; cognitive behavioural therapy (CBT) [9]; and exposure treatment [10]. While patients and clients may be discussing this form of anxiety in personal therapy, we consider this is unlikely, and, in any case, it is not reported in the psychotherapeutic literature. A search of the Psychoanalytic Electronic Publishing database (comprising over 100,000 articles) returned only one match on the subject and that being from the 1940s [11]. 
EMDR has gained empirical support in the areas of posttraumatic stress disorder (PTSD) and anxiety across the lifespan and is recommended by the UK's National Institute of Clinical Excellence (NICE) as an empirically supported PTSD and phobia treatment. Research applying EMDR to dental anxiety is however sparse, though two reports in the literature on a limited number of patients promisingly showed the efficacy of EMDR in reducing dental anxiety in patients undergoing dental treatments $[6,12]$. Moreover, NICE is arguably biased against therapeutic approaches other than CBT, i.e., those based on psychoanalytic/psychodynamic and humanistic therapies, so we do not have any robust evidence of possible comparative therapeutic intervention efficacy or otherwise.

\section{Future Directions}

As a result of this mini-review, planned research will be undertaken in two phases, both of which will be examples of interdisciplinary professionals working in the health sector. This is not only promoted at our own university, the Auckland University of Technology (AUT), but also through a review by the Health and Disability Commissioner [13] which identified that many complaints to the Commissioner arise from failures in teamwork across professions, and hence all New Zealand health care practitioners are obligated to practice collaboratively in order to improve health outcomes as enshrined (from 2019) in the Health Practitioners Competence Assurance Amendment Act [14].

The first phase is a scoping review, in effect, a "mapping" of key nonpharmacological/therapeutic responses to dental anxiety, first globally, and then nationally with regard to their availability in Aotearoa New Zealand. According to Wilson et al., scoping reviews map important ideas supporting a research area and the main sources and types of evidence available [15]. As this includes locating a varied set of sources, such as qualitative research studies, textbooks, reports, and what is referred to as "grey" literature, this analysis aligns with the aim of this project to identify and map the "scope" of literature in the broader sense than that of a systematic review [16]. This phase will commence with the joint supervision of an undergraduate Summer studentship.

The second phase will compare the efficacy of different therapeutic interventions such as hypnotherapy, EMDR, CBT and other, more generic psychotherapeutic approaches in reducing or curing dental anxiety.

\section{Conclusion}

Dental anxiety is a major barrier to dental services and considering it is a complex health issue, will require well- coordinated interprofessional collaboration to inform treatment. Increased uptake in dental care can lead to an increase in oral health in the population.

\section{References}

1. Neumann J, Schroeder H, Voss P (1989) Mental health and wellbeing in the context of the health promotion concept. In: Neumann J, Schroeder $\mathrm{H}$, Voss P (Eds.), Mental health within the health promotion concept. Dresden, Germany: German Hygiene Museum/Copenhagen: WHO, Geneva, Switzerland, pp. 3-17.

2. World Health Organisation (2014) Mental health: Strengthening our response.

3. Peres MA, Macpherson LMD, Weyant RJ, Daly B, Venturelli B, et al. (2019) Oral diseases: a global public health challenge. Lancet 394(10194): 249260.

4. Ibrahim H, Lyons KM, Armfield JM, Thomson WM (2017) Performance of the index of dental anxiety and fear in a population-based sample of adults. Aust Dent J 62(4): 478-484.

5. Locker D, Poulton R, Thomson WM (2001) Psychological disorders and dental anxiety in a young adult population. Community Dent Oral Epidemiol 29(6): 456-463.

6. Freeman R (1985) Dental anxiety: a multifactoral aetiology. Br Dent J 159(12): 406-408.

7. Armfield JM, Heaton LJ (2013) Management of fear and anxiety in the dental clinic: a review. Aust Dent J 58(4): 390-407.

8. De Jongh A, van den Oord HJM, Ten Broeke E (2002) Efficacy of eye movement desensitisation and reprocessing in the treatment of specific phobias: Four single case studies on dental phobia. J Clin Psychol 58(12): 1489-1503.

9. Davies JG, Wilson KI, Clements AL (2011) A joint approach to treating dental phobia: A re-evaluation of a collaboration between community dental services and specialist psychotherapy services ten years on. $\mathrm{Br}$ Dent J 211(4): 159-162.

10. Arias MC, McNeil DW (2020) Smartphone-based exposure treatment for dental phobia: A pilot randomised clinical trial. J Public Health Dent 80(1): 23-30.

11. Coriat IH (1946) Dental anxiety: Fear of going to the dentist. Psychoanal Rev 33: 365-367.

12. Lu DP (2010) Using alternating bilateral stimulation of eye movement desensitisation for treatment of fearful patients. Gen Dent 58(3): e140147.

13. Health and Disability Commissioner (2012) 2012 Review of the Health Practitioners Competence Assurance Act 2003. New Zealand.

14. New Zealand Government (2019) Health Practitioners Competence Assurance Amendment Act 2019. New Zealand.

15. Wilson M, Lavis J, Guta A (2012) Community-based organisations in the health sector: A scoping review. Health Res Policy Syst 10: 36.

16. Levac D, Colquhoun H, O’Brien KK (2010) Scoping studies: Advancing the methodology. Implement Sci 5: 69. 